\title{
APPARENT HORIZONS IN CLIFTON-MOTA-BARROW INHOMOGENEOUS UNIVERSE
}

\author{
VINCENZO VITAGLIANO ${ }^{1}$, VALERIO FARAONI ${ }^{2}$, THOMAS P. SOTIRIOU \\ and STEFANO LIBERATI ${ }^{3}$ \\ 1 CENTRA, Departamento de Física, Instituto Superior Técnico, Universidade Técnica de \\ Lisboa - UTL, Av. Rovisco Pais 1, 1049 Lisboa, Portugal \\ 2 Physics Department and STAR Research Cluster, Bishop's University, \\ Sherbrooke, Québec, Canada J1M $1 Z^{7}$ \\ 3 SISSA, Via Bonomea 265, 34136 Trieste, Italy and \\ INFN, Sezione di Trieste, Italy
}

\begin{abstract}
We analyze the apparent horizon dynamics in the inhomogeneous Clifton-MotaBarrow solution of Brans-Dicke theory. This solution models a central matter configuration embedded in a cosmological background. In certain regions of the parameter space we find solutions exhibiting dynamical creation or merging of two horizons.
\end{abstract}

\section{Introduction}

Scalar-tensor theories lead to a spacetime-dependent effective gravitational coupling $G_{\text {eff. }}$ In theories with this characteristic, the problem of understanding the behaviour of a local system embedded in a cosmological environment (cf. the McVittie solution $^{1}$ of general relativity which is not yet well understood), is of particular interest. This motivated the study of inhomogeneous analytical solutions of scalar-tensor theories of gravity.

The Clifton-Mota-Barrow spherically symmetric and time-dependent metric ${ }^{2}$ is

$$
d s^{2}=-A(\varrho)^{2 \alpha} d t^{2}+a^{2}(t)\left[\left(1+\frac{m}{2 \alpha \varrho}\right)^{4} A(\varrho)^{\frac{2}{\alpha}(\alpha-1)(\alpha+2)}\right]\left(d \varrho^{2}+\varrho^{2} d \Omega^{2}\right),
$$

with

$$
A(\varrho)=\frac{1-\frac{m}{2 \alpha \varrho}}{1+\frac{m}{2 \alpha \varrho}}, \quad \alpha=\sqrt{\frac{2\left(\omega_{0}+2\right)}{2 \omega_{0}+3}}, \quad a(t)=a_{0}\left(\frac{t}{t_{0}}\right)^{\frac{2 \omega_{0}(2-\gamma)+2}{3 \omega_{0} \gamma(2-\gamma)+4}} \equiv a_{*} t^{\beta}
$$

where $\gamma-1$ is the barotropic index of the equation of state for the cosmological perfect fluid. This metric is an explicit solution of Brans-Dicke theory, which is described by the action

$$
S_{B D}=\int d^{4} x \sqrt{-g}\left[\phi R-\frac{\omega_{0}}{\phi} g^{\mu \nu} \nabla_{\mu} \phi \nabla_{\nu} \phi+\mathcal{L}^{(m)}\right],
$$

where the effective gravitational coupling is proportional to the inverse of the scalar field $\phi(\varrho, t)$. The constant $\omega_{0}$ is often called the Brans-Dicke parameter.

We would like to understand the dynamics of horizons (black hole and cosmological) in the Clifton-Mota-Barrow solution. Given that the solution is asymptotically Friedman-Lemaître-Robertson-Walker (FLRW) and dynamical, the most straightforward way to do so is to look for apparent horizons (note, however, potential caveats in the use for apparent horizon, as they depend on the foliation ${ }^{3,4}$ ). An 
apparent horizon (elsewhere dubbed "trapping horizon" 5 ) is a space- (or time-)like surface defined as the closure of a surface foliated by marginally trapped surfaces.

What follows is based on the analysis and results of Ref. 6 .

\section{Location of the apparent horizons}

The existence and location of the apparent horizons are determined by the condition $g^{r r}=0$, where $r$ is the areal radius (not to be confused with the isotropic radius $\varrho)$. For the Clifton-Mota-Barrow solutions and for an expanding universe with $H \equiv \dot{a} / a>0$ this condition reads ${ }^{6}$

$$
H r^{2}-\frac{(\alpha-1)(\alpha+2)}{\alpha^{2}} \operatorname{ma}(t) A(r)^{\frac{2(\alpha-1)(\alpha+1)}{\alpha}}-A(r)^{\alpha+1} r=0,
$$

where $H=\beta / t$ is the Hubble parameter corresponding to the scale factor $a(t)$.

The scalar curvature is singular in the limit $r \rightarrow 0$, denoting the presence of a central singularity. Once the variable $x \equiv \frac{m}{2 \alpha \varrho}$ has been introduced, eq. (1) can be solved parametrically for the radius $r$ of the apparent horizon(s) and the time coordinate $t$,

$$
\begin{aligned}
r(x) & =a_{*} t^{\beta} \frac{m}{2 \alpha} \frac{(1+x)^{2}}{x}\left(\frac{1-x}{1+x}\right)^{\frac{(\alpha-1)(\alpha+2)}{\alpha}}, \\
t(x) & =\left\{\frac{2 \alpha}{m a_{*} \beta} \frac{x}{(1+x)^{\frac{2}{\alpha}(\alpha+1)}}\left[(1-x)^{2 / \alpha} 2 x \frac{(\alpha-1)(\alpha+2)}{\alpha}(1-x)^{-2(\alpha-1) / \alpha}\right]\right\}^{\frac{1}{\beta-1}} .
\end{aligned}
$$

As a typical example, we plot the radius of the apparent horizon as a function of time (in adapted units) for the case $\omega_{0}=1$. The red (dashed) curve is for dust $\left(\gamma_{D}=1\right)$, the green (solid) curve is for both radiation and stiff matter $\left(\gamma_{R}=4 / 3\right.$ and $\gamma_{S M}=2$ ), while the blue (dotted) one corresponds to a cosmological constant $\left(\gamma_{\Lambda}=0\right)$. The initial behaviour (a unique, expanding apparent horizon) is common in all of these different configurations.

For dust, radiation, and stiff matter, two further horizons appear at a certain time $t_{*}$, the outermost of which expands forever, while the other merges with the initial horizon, leaving behind a naked singularity covered by a cosmological horizon (cf. the contribution by V. Faraoni to these Proceedings, a similar solution by Husain, Martinez, and Nunez, ${ }^{7}$ and its interpretation ${ }^{8}$ ). The cosmological constant case is different in the fact that the two horizons appear inside the initial one, as shown in the right panel of Fig. 1.

The complete analysis of the Clifton-Mota-Barrow solution reveals a richer phenomenology ${ }^{6}$ than just the previous example. In particular, the general relativity limit $\omega_{0} \rightarrow \infty$ turns out to correspond to a generalized McVittie metric. ${ }^{9}$ In this case an initial naked singularity is then covered by two appearing horizons. This is true for any equation of state of the cosmological fluid except for the cosmological constant. For the latter the reverse happens, that is, an expanding and a contracting horizon merge to leave behind a naked singularity. 

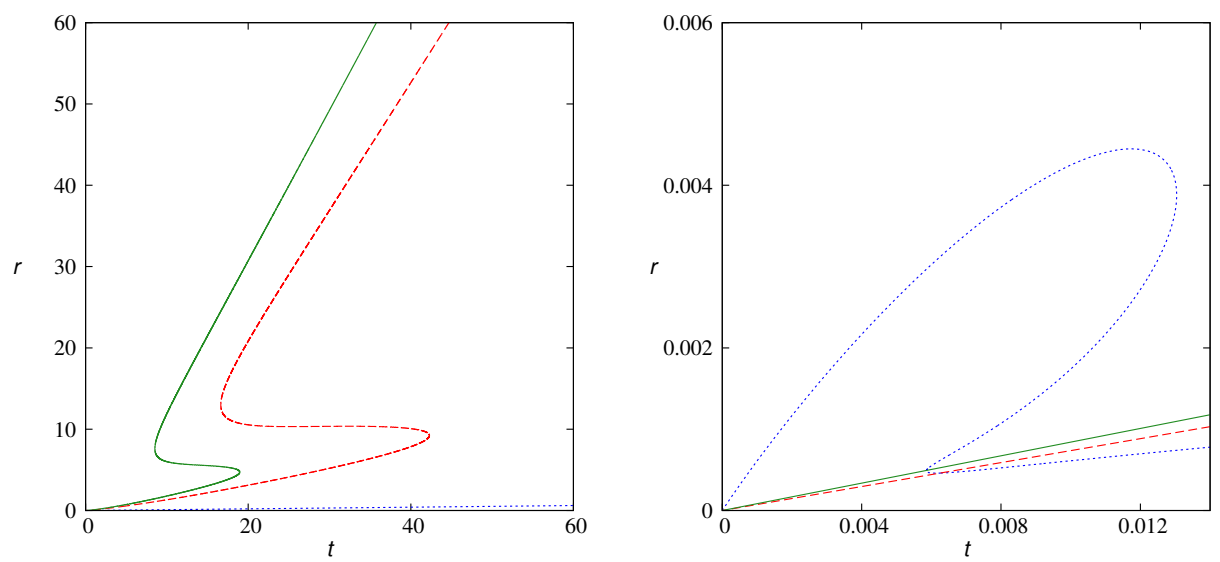

Fig. 1. Radius of the apparent horizon as function of time in adapted units, for $\omega_{0}=1$. The right panel focuses on the early time region of the plot.

We conclude with a warning: as already mentioned, apparent horizons depend on the spacetime slicings adopted, namely, specific slicings could imply the absence of apparent horizons even though the singularity is hidden by an event horizon. From this perspective, the issue of the presence of the naked spacetime singularity reported above becomes particularly delicate. Nonetheless, looking for apparent horizons is probably the most straightforward probe for event horizon and it seems unlikely that slicing dependence seriously affects the results for a spherically symmetric foliation of a FLRW spacetime.

\section{Acknowledgements}

VV is supported by FCT - Portugal through the grant SFRH/BPD/77678/2011. VF would like to thank NSERC for financial support. TPS acknowledges financial support provided under the Marie Curie Career Integration Grant LIMITSOFGR2011-TPS and the European Union's FP7 ERC Starting Grant "Challenging General Relativity" CGR2011TPS, grant agreement no. 306425 .

\section{References}

1. G. C. McVittie, Mon. Not. Roy. Astron. Soc. 93, 325 (1933).

2. T. Clifton, D. F. Mota and J. D. Barrow, Mon. Not. Roy. Astron. Soc. 358, 601 (2005).

3. R. M. Wald and V. Iyer, Phys. Rev. D 44, 3719 (1991).

4. E. Schnetter and B. Krishnan, Phys. Rev. D 73, 021502 (2006).

5. S. A. Hayward, Phys. Rev. D 49, 6467 (1994).

6. V. Faraoni, V. Vitagliano, T. P. Sotiriou and S. Liberati, Phys. Rev. D 86, 064040 (2012).

7. V. Husain, E. A. Martinez and D. Nunez, Phys. Rev. D 50, 3783 (1994).

8. V. Faraoni and A. F. Zambrano Moreno, Phys. Rev. D 86, 084044 (2012).

9. V. Faraoni, C. Gao, X. Chen and Y. -G. Shen, Phys. Lett. B 671, 7 (2009). 